\section{Fast block exact Gauss-Seidel pseudo affine projection algorithm}

\section{F. Albu and H.K. Kwan}

\begin{abstract}
A block affine projection algorithm that is less complex but mathematically equivalent to a recently proposed Gauss-Seidel pseudo affine projection (GS-PAP) algorithm is proposed. This reduced complexity is achieved by applying a partitioning method to the original sampleby-sample algorithm. It is shown that the derived algorithm has better convergence and tracking abilities than those of the NLMS algorithm. Its application in an acoustic echo cancellation is investigated.
\end{abstract}

Introduction: Adaptive filtering is essential in applications such as system identification, channel equalisation, active noise control, acoustic echo cancellation, etc. The well-known normalised LMS (NLMS) algorithm has been widely used but it has a slow asymptotic convergence. The affine projection (AP) algorithm [1] can be considered as a generalisation of the NLMS algorithm. Although too complex for most applications, it provides a much improved convergence speed compared to that of the NLMS. Many other affine projection algorithms have been proposed (see, e.g. [2-5] and the references therein). The sample-by-sample algorithms [2-3] are still complex for some applications such as acoustic echo cancellation. Subband or block processing variants [4-5] have much reduced complexity but it is still desirable to have a uniform distribution of the computations for an efficient implementation. In this Letter, a novel and efficient algorithm called the fast block exact Gauss-Seidel pseudo affine projection algorithm (FBEGS-PAP) is derived.

Let us take the following notations: $x(n)$ is the input signal and $y(n)$ is the desired output signal. $e(n)$ is the output error and $\bar{e}(n)$ is the normalised error. $\mathbf{X}(n)=[x(n), \ldots, x(n-L+1)]^{T}$ where $L$ is the filter length. $R(n)$ is the autocorrelation matrix of the signal. $\xi(n)=[x(n), \ldots$, $x(n-N+1)]^{T}$ where $N$ is the affine projection order. $\delta$ is a regularisation factor. $\mu$ is the step size. $\mathbf{U}(n)=[u(n), \ldots, u(n-L+1)]^{T}$ is the approximated decorrelation vector. $\mathbf{b}$ is an $N$ vector with only one nonzero element that is unity at the top. $\mathbf{H}(n)=\left[h_{1}(n), \ldots, h_{L}(n)\right]^{T}$ is the filter coefficients vector. $\mathbf{P}$ is an $N$ length vector and $P_{i}, i=0$ to $N-1$ is its $i$ th element. Below are the equations of the derived Gauss-Seidel pseudo affine projection algorithm (GS-PAP) [2]:

$$
\text { Initialisations: } \begin{aligned}
\mathbf{X}(-1) & =\mathbf{0}, \mathbf{R}(-1)=\delta \mathbf{I}, \mathbf{P}(-1) \\
& =\mathbf{b} / \delta, \mathbf{U}(-1)=\mathbf{0}, \mathbf{H}(-1)=\mathbf{0}
\end{aligned}
$$

At each sample $n \geq 0$,

$$
\mathbf{R}(n)=\mathbf{R}(n-1)+\xi(n) \xi^{T}(n)-\xi(n-L) \xi^{T}(n-L)
$$

$\mathbf{R}(n) \mathbf{P}(n)=\mathbf{b}$ (can be solved by the Gauss-Seidel method)

$$
\begin{aligned}
e(n) & =y(n)-\mathbf{X}^{T}(n) \mathbf{H}(n-1) \\
\mathbf{U}(n) & =\frac{1}{P_{0}(n)} \sum_{i=0}^{N-1} P_{i}(n) \mathbf{X}(n-i) \\
\bar{e}(n) & =\frac{\mu}{\mathbf{U}^{T}(n) \mathbf{U}(n)+\delta} e(n) \\
\mathbf{H}(n) & =\mathbf{H}(n-1)+\mathbf{U}(n) \bar{e}(n)
\end{aligned}
$$

It can be seen that (6) and (7) lead to a similar update equation as the NLMS algorithm. The basic idea of reducing complexity in the GS-PAP adopted in this Letter is similar to that used in [6] for deriving the modified fast exact NLMS (MFENLMS) algorithm. Denote $M$ as the block size. We partition the vectors $\mathbf{X}(n), \mathbf{U}(n)$ and $\mathbf{H}(n)$ according to:

$$
\begin{aligned}
& \mathbf{X}^{T}(n)=\left[\mathbf{X}_{a}^{T}(n) \mathbf{X}_{b}^{T}(n-2 M)\right], \quad \mathbf{U}^{T}(n)=\left[\mathbf{U}_{a}^{T}(n) \mathbf{U}_{b}^{T}(n-2 M)\right], \\
& \mathbf{H}^{T}(n)=\left[\mathbf{H}_{a}^{T}(n) \mathbf{H}_{b}^{T}(n)\right] \text { with } \quad \mathbf{X}_{a}^{T}(n)=[x(n), \ldots, x(n-2 M+1)], \\
& \mathbf{U}_{a}^{T}(n)=[u(n), \ldots, u(n-2 M+1)], \quad \mathbf{H}_{a}^{T}(n)=\left[h_{1}(n), \ldots, h_{2 M}(n)\right], \\
& \mathbf{X}_{b}^{T}(n)=[x(n-2 M), \ldots, x(n-L+1)] \text {, } \\
& \mathbf{U}_{b}^{T}(n)=[u(n-2 M), \ldots, u(n-L+1)], \\
& \mathbf{H}_{b}^{T}(n)=\left[h_{2 M+1}(n), \ldots, h_{L}(n)\right]
\end{aligned}
$$

The equations (4) to (7) of the GS-PAP algorithm can be partitioned into an 'updating' part and a 'fixed' filtering part as in [6]. Computation of the 'updating' part with a lower order of complexity is performed at each recursion step, whereas the 'fixed' part can be computed from $M$ recursion steps. Therefore, the non-uniformly distribution of the arithmetic operations within a block of $M$ samples, encountered in other block algorithms [5-6], can be avoided.

Equation (7) is repeatedly substituted in (4) taking into account the previous partitions. The following equations are obtained for $i=n, \ldots, n-M+1$ :

$$
\begin{aligned}
& \hat{y}_{a}(i)=\mathbf{X}_{a}^{T}(i) \mathbf{H}_{a}^{T}(i)+\sum_{j=1}^{M+i-n} \sigma_{j}(i-2 M) \bar{e}(i-j) \\
& \sigma_{j}(i-2 M)=\mathbf{U}_{b}^{T}(i-2 M-j) \mathbf{X}_{b}(i-2 M) \\
& \hat{y}_{b}(n-M)=\mathbf{U}_{b}^{T}(i-2 M) \mathbf{H}_{b}(n-M) \\
& \mathbf{H}_{a}(i)=\mathbf{H}_{a}(i-1)+\mathbf{X}_{a}(i-1) \bar{e}(i-1) \\
& \mathbf{H}_{b}(n)=\mathbf{H}_{b}(n-M)+\sum_{j=1}^{L} \mathbf{U}_{b}(n-2 M-j) \bar{e}(n-j) \\
& e(i)=y(i)-\hat{y}_{a}(i)-\hat{y}_{b}(n-M)
\end{aligned}
$$

The fast block exact GS-PAP (FBEGS-PAP) algorithm includes (2)-(3), (5)-(6), (8)-(13). All the equations excepting (10) and (12) are performed every step. Their complexity is small because usually $M$ and $N$ are much smaller than the filter length $L$. The 'filtering part' ((10) and (12)) depends on the data from the last block. This part involves the computation of $M$ successive outputs of a fixed coefficient filter. Several fast FIR filtering procedures that reduce significantly the number of operations exist (e.g. based on linear or circular convolution). We used the efficient FIR filtering architecture presented in Fig. 2 of [7] for cases when the block size is a power of two $\left(M=2^{k}\right)$.

The numerical complexities of the algorithms are measured by counting the number of multiplications and divisions per recursion. Therefore we obtain: $C_{N L M S}=2 L+4 ; C_{M F E N L M S}=10 M+1+\left[2(3 / 4)^{k}(L-2 M)\right]$;

$$
\begin{aligned}
C_{F B E G S-P A P} & =10 M+2+\left[2(3 / 4)^{k}(L-2 M)\right]+N^{2}+4 N \\
C_{G S-P A P} & =2 L+N^{2}+3 N+5
\end{aligned}
$$

The increase in complexity of the FBEGS-PAP algorithm in comparison with that of the MFENLMS is rather small for the usual values of $N$ and does not depend on $L$ or $M$. The proper choice of the block size is of crucial importance in reducing the number of multiplies and divisions. As shown in [6], there is an 'optimal' block size value of $M$ that minimises its complexity if the projection order is fixed. It can be easily deduced that the value of the block size that minimises the number of multiplies and divisions is higher for a higher filter length (e.g. if $N=10$, the 'optimal' value of $M$ is 8 for $L=256$, and is 32 for $L=1024$, etc.).

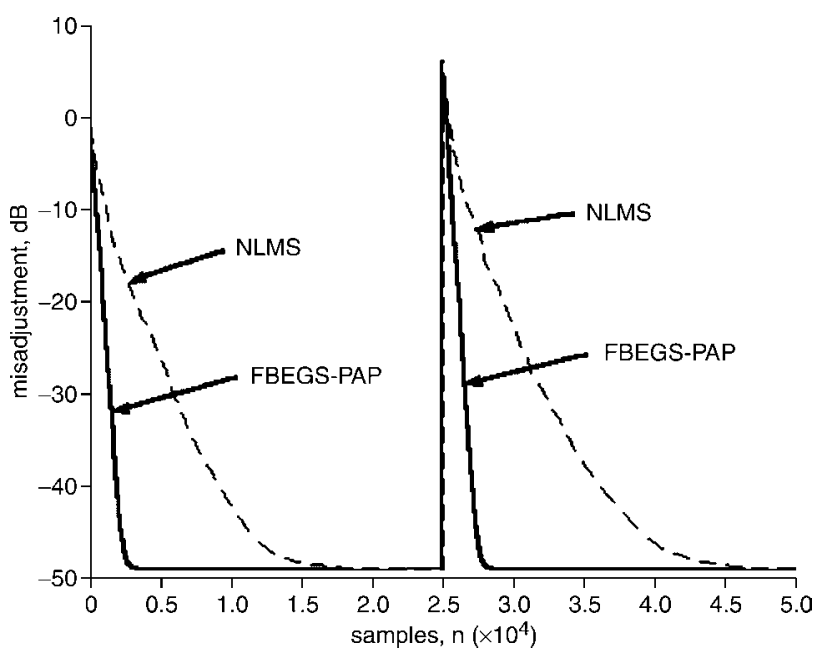

Fig. 1 Learning curves of FBEGS-PAP and NLMS algorithms for coloured excitation under sudden change in echo path

$(L=256, N=10, M=8, \mu=1)$

Results: We tested the convergence and tracking abilities of the FBEGS-PAP algorithm using coloured noise and speech (white noise has also been used but not shown here) as excitation signals in an acoustic echo cancellation example. As expected, because it is mathematically equivalent to the GS-PAP, it inherits its performances. An example of the superior convergence and tracking abilities of the 
FBEGS-PAP algorithm as compared to the NLMS when using a coloured excitation signal is shown in Fig. 1. In our simulations, we used $L=256, N=10, M=8, \mu=1$. The impulse response of the measured car cabin impulse response was truncated to 259 coefficients, so that the theoretical minimum misalignment was calculated to be $-49.06 \mathrm{~dB}$. The tracking abilities of the algorithms were investigated by a sudden change in the sign of the measured echo path coefficients after 25000 samples. The convergence of the algorithms was compared using the squared norm of the difference between the car cabin impulse response and the adaptive filter (in dB). The FBEGS-PAP algorithm was stable in all our 32-bit floatingpoint simulations and the learning curves were virtually identical with those of the GS-PAP in double precision simulations (Fig. 1). Table 1 compares the numerical complexity of the considered algorithms for different filter lengths, block sizes and projection orders. It can be seen that the complexity of the FBEGS-PAP is significantly reduced in comparison with those of the NLMS and the GS-PAP. Also, it is marginally more complex than that of the MFENLMS for moderate values of the affine projection order (see Table 1).

Table 1: Computational complexity (number of multiplications and divisions per recursion) of investigated algorithms for different filter lengths, $L$, block sizes, $M$, and projection orders, $N$

\begin{tabular}{|c|c|c|c|c|}
\hline \multirow{2}{*}{ Algorithm } & \multicolumn{2}{|c|}{$L=256, M=8$} & \multicolumn{2}{c|}{$L=1024, N=10$} \\
\cline { 2 - 5 } & $N=10$ & $N=4$ & $M=32$ & $M=8$ \\
\hline FBEGS-PAP & $\mathbf{4 2 5}$ & $\mathbf{3 1 7}$ & $\mathbf{9 1 8}$ & $\mathbf{1 0 7 3}$ \\
\hline GS-PAP [2] & 647 & 545 & 2183 & 2183 \\
\hline MFENLMS [6] & 284 & 284 & 777 & 932 \\
\hline NLMS & 516 & 516 & 2052 & 2052 \\
\hline
\end{tabular}

Conclusion: We have proposed an efficient implementation of the GS-PAP algorithm. It was proved that the FBEGS-PAP algorithm has significant better performances and reduced complexity in comparison with those of the NLMS. Its stability in 32-bit floating-point operations and uniform distribution of operations make it attractive for applications in acoustic echo cancellation systems.

(C) IEE 2004

22 July 2004

Electronics Letters online no: 20046320

doi: 10.1049/el:20046320

F. Albu (Department of Telecommunications, Faculty of Electronics and Telecommunications, 'POLITEHNICA', University of Bucharest, 1-3 Bd. Iuliu Maniu, Sector 6, Complex Leu, Building B, Bucharest, B117, Romania)

E-mail: felix_albu@ieee.org

H.K. Kwan (Department of Electrical and Computer Engineering, University of Windsor, 401 Sunset Avenue, Windsor, Ontario N9B 3P4, Canada)

\section{References}

1 Ozeki, K., and Umeda, T.: 'An adaptive filtering algorithm using an orthogonal projection to an affine subspace and its properties', Electron. Commun. Jpn, 1984, 67-A, (5), pp. 19-27

2 Albu, F., and Kwan, H.K.: 'Combined echo and noise cancellation based on Gauss-Seidel pseudo affine projection algorithm', Proc. IEEE Int. Symp. on Circuits and Systems, ISCAS 2004, Vancouver, Canada, May 2004, pp. 505-508

3 Bouchard, M.: 'Multichannel affine and fast affine projection algorithms for active noise control and acoustic equalization systems', IEEE Trans. Speech Audio Process., 2003, 11, (1), pp. 54-60

4 Liu, Q.G., Champagne, B., and Ho, K.C.: 'On the use of a modified fast affine projection algorithm in subbands for acoustic echo cancellation', IEEE Digital Signal Processing Workshop Proc., 1996, Loen, Norway, September 1996, pp. 354-357

5 Robouts, G., and Moonen, M.: 'A sparse block exact affine projection algorithm', IEEE Trans. Speech Audio Process., 2002, 10, (2), pp. 100-108

6 Schutze, H., Ren, Z., and Hartmann, I.: 'Modified fast exact NLMS adaptive algorithm', Electron. Lett., 1994, 30, pp. 1029-1031

7 Kwan, H.K., and Tsim, M.T.: 'High speed 1-D FIR digital filtering architectures using polynomial convolution', Proc. IEEE Int. Conf. on Acoustics, Speech, and Signal Processing, Dallas, TX, USA, April 1987, pp. $1863-1866$ 